\title{
Further insights into the eco-epidemiology of American cutaneous leishmaniasis in the Belem metropolitan region, Pará State, Brazil
}

\author{
Lucas Pantoja Gonçalves ${ }^{[1]}$, Thiago Vasconcelos dos Santos ${ }^{[1]}$, \\ Marliane Batista Campos ${ }^{[1]}$, Luciana Vieira do Rêgo Lima ${ }^{[1]}$, Edna Aoba Yassui Ishikawa ${ }^{[2]}$, \\ Fernando Tobias Silveira ${ }^{[1],[2]}$ and Patrícia Karla Santos Ramos ${ }^{[1]}$
}

[1]. Instituto Evandro Chagas, Laboratório de Leishmanioses 'Prof. Dr. Ralph Lainson', Seção de Parasitologia, Ananindeua, PA, Brasil.

[2]. Universidade Federal do Pará, Núcleo de Medicina Tropical, Belém, PA, Brasil.

\begin{abstract}
Introduction: In the Belém Metropolitan Region (BMR), Pará State, Brazil, American cutaneous leishmaniasis (ACL) is endemic; however, very little is known regarding its causative agents. Therefore, we used our standard diagnostic approach combined with an RNA polymerase II largest subunit (RNAPOIILS)-polymerase chain reaction (PCR) followed by analysis of restriction fragment length polymorphism (PCR-RFLP) to identify Leishmania spp. ACL agents in this region. Methods: Thirty-two Leishmania spp. isolates from patients with ACL in the BMR during 1995-2018 were analyzed. Leishmania spp. DNA samples were amplified using the primers RPOR2/RPOF2, and the 615-bp PCR products were subjected to enzymatic digestion using TspRI and HgaI endonucleases. Results: ACL etiological agents in the BMR comprised Leishmania (Viannia) lindenbergi (43.7\%) followed by Leishmania (Viannia) lainsoni (34.4\%), Leishmania (Leishmania) amazonensis (12.5\%), and Leishmania (Viannia) braziliensis (9.4\%). Conclusions: To our knowledge, the results of the study revealed for the first time that $L$. (V.) lindenbergi and $L .(V$.) lainsoni are the main ACL agents in BMR.
\end{abstract}

Keywords: American cutaneous leishmaniasis. Leishmania spp. Molecular characterization. PCR-RFLP. Amazonian Brazil.

\section{INTRODUCTION}

American cutaneous leishmaniasis (ACL) is a parasitic protozoan disease caused by different Leishmaniinae parasites (Kinetoplastida: Trypanosomatidae) and is widely distributed in most Latin American countries. There are at least 15 recognized species within the subgenera Leishmania (Leishmania), L. (Viannia), and L. (Mundinia) that may give rise to human diseases ${ }^{1,2}$. Seven well-known Leishmania species have been identified as ACL agents in Brazil; Leishmania (V.) braziliensis, L. (V.) guyanensis, L. (V.) lainsoni, L. (V.) shawi, L. (V.) naiffi, L. (V.) lindenbergi, and $L$. (L.) amazonensis ${ }^{3}$. More recently, a new subspecies, $L$. (V.) shawi santarensis, as well as the first hybrid parasite, $L$. (V.) guyanensis/ L. (V.) shawi shawi, have been found in the Brazilian Amazon ${ }^{4}$.

\footnotetext{
Corresponding author: Dra. Patrícia Karla Santos Ramos.

e-mail: patriciaramos@iec.gov.br

(1) https://orcid.org/0000-0002-0275-0994

Received 22 May 2020

Accepted 19 October 2020
}

ACL behaves as a primary zoonosis of wild mammals in Brazil, and the transmission of Leishmania species occurs through the bites of infected females of different phlebotomine vectors (Diptera: Psychodidae $)^{5-8}$. ACL has an occasional but endemic character in the Belém Metropolitan Region (BMR), Pará State, in the Brazilian Amazon that is mainly associated with three Leishmania species, including $L$. (L.) amazonensis ${ }^{9}, L$. (V.) lainsoni $^{10}$, and $L$. $\left(V\right.$ ) lindenbergi $i^{11}$. Over the years, the BMR has experienced an increase in growth rate with intense urban construction and displacement of populations to areas neighboring secondary native forest areas, favoring human contact with the enzootic cycles of these Leishmania species.

The identification of potential ACL agents is a key step in surveillance strategies, and the existing knowledge and molecular tools available for the identification and characterization of Leishmania species must be improved and harmonized ${ }^{12}$. In this sense, species typing has evolved into a molecular approach. An overview of the different methods and targets currently available can be found elsewhere ${ }^{13}$. 
Polymerase chain reaction (PCR) followed by restriction fragment length polymorphism (RFLP) has been widely applied to characterize New World Leishmania species, and has been focused on different targets of kinetoplast or genomic DNA ${ }^{14-19}$. Due to the high inter/intraspecific diversity/polymorphism in the parasites, these techniques do not usually show continental reproducibility, and regional-scale assays must be improved to validate established protocols. To this end, a set of targets that encode the genes of the RNA polymerase II largest subunit (RNAPOIILS; considered phylogenetically informative as defined by parsimony criteria) has been used to explore the relationships between Leishmania species $^{20}$. We used our standard diagnostic approach combined with the RNAPOIILS-PCR-RFLP assay (previously designed to identify Amazonian/Guianan Leishmania species) ${ }^{19}$ to identify Leishmania spp. that act as potential ACL agents in the BMR. These results provide crucial new insights into the eco-epidemiology of ACL in this region.

\section{METHODS}

\section{Study area}

The BMR comprises a cluster of socioeconomic integrated municipalities located in the northeastern Pará State, Brazil (Belém [the State capital], Ananindeua, Marituba, Benevides, Santa Izabel do Pará, Santa Bárbara do Pará, and Castanhal), with a resident population of approximately 2,505,242 inhabitants ${ }^{21}$ and a territorial area of $6.890 \mathrm{~km}^{2}$ (Figure 1). The landscape has an extensive alluvial plain, with a typically equatorial climate and average temperatures ranging from $24^{\circ} \mathrm{C}$ to $26^{\circ} \mathrm{C}$ and humidity above $80 \%$. The annual precipitation is approximately $2500 \mathrm{~mm}$, with a rainy season from January to June. The vegetation is mainly secondary forest, although some original remnants still cover $\sim 31 \%$ of the region, which is composed of upland (terra firme), floodplain (várzea), and wetland (igapó) forests ${ }^{22}$.

\section{Surveying the ACL epidemiology in the BMR}

Patients with ACL examined at the Ralph Lainson Leishmaniases Lab (with the BMR as the geographical local of presumed infection from 1995 to 2018) were also screened following our standard diagnostic approach comprising clinical-epidemiological investigation and laboratory diagnosis. The patients were diagnosed by parasitological demonstrations (Giemsa-stained smears of exudates from cutaneous lesions) and by the interpretation of the Montenegro skin test (inactivated promastigotes of $L$. $(V$.) braziliensis $-\mathrm{MHOM} / \mathrm{BR} / \mathrm{M} 17323-1 \times 10^{7}$ parasites $/ \mathrm{mL}$ ), as previously described ${ }^{4,23,24}$. In vitro/in vivo parasite isolation (inoculating exudates from cutaneous lesions in Difco $\mathrm{B}^{45}$ media and/or in the hind foot of Mesocricetus auratus) was also performed routinely ${ }^{25}$. The ACL-confirmed cases received systemic therapy (meglumine antimoniate) at a dose of $12 \mathrm{mg} \mathrm{Sb5} / \mathrm{kg} /$ day in two series of 22 days, with an interval of 7-10 days between each series ${ }^{23}$.

All Leishmania spp. isolates obtained from ACL cases of localized cutaneous leishmaniasis (LCL) clinical form originating from the BMR from 1995 to 2018 were included in the analysis. Of the 32 cultured samples, 16 were from the municipality of Belém, seven from Ananindeua, four from Benevides, three from Santa Izabel, one from Santa Bárbara do Pará, and one from Castanhal. No isolate was registered in the municipality of Marituba (Figure 1).

\section{DNA extraction/quantification}

DNA was extracted from positive culture samples using the commercial Reliaprep gDNA Tissue Miniprep System Kit (Promega, USA). After performing cell lysis using proteinase K, the samples were placed in a column surrounded by a silica membrane, and during centrifugation, the DNA adhered to the membrane. After several washes, the extracted DNA was eluted in $150 \mu \mathrm{L}$ of elution buffer. DNA sample quantification was performed using a Qubit 2.0 fluorometer (Invitrogen, USA).

\section{RNAPOIILS-PCR-RFLP}

The methodology was adapted from Simon et al. ${ }^{19}$ with minor modifications. In brief, Leishmania DNA amplification was performed using the primers RPOF2 (5'-AGAACATGGGCGGCC-3') and RPOR2 (5'-CGAGGGTCACGTTCTTG-3'), which amplified a 615-bp fragment of the RNAPOIILS gene. The reaction was performed in a final volume of $50 \mu \mathrm{L}$ containing $0.2 \mu \mathrm{M}$ of each

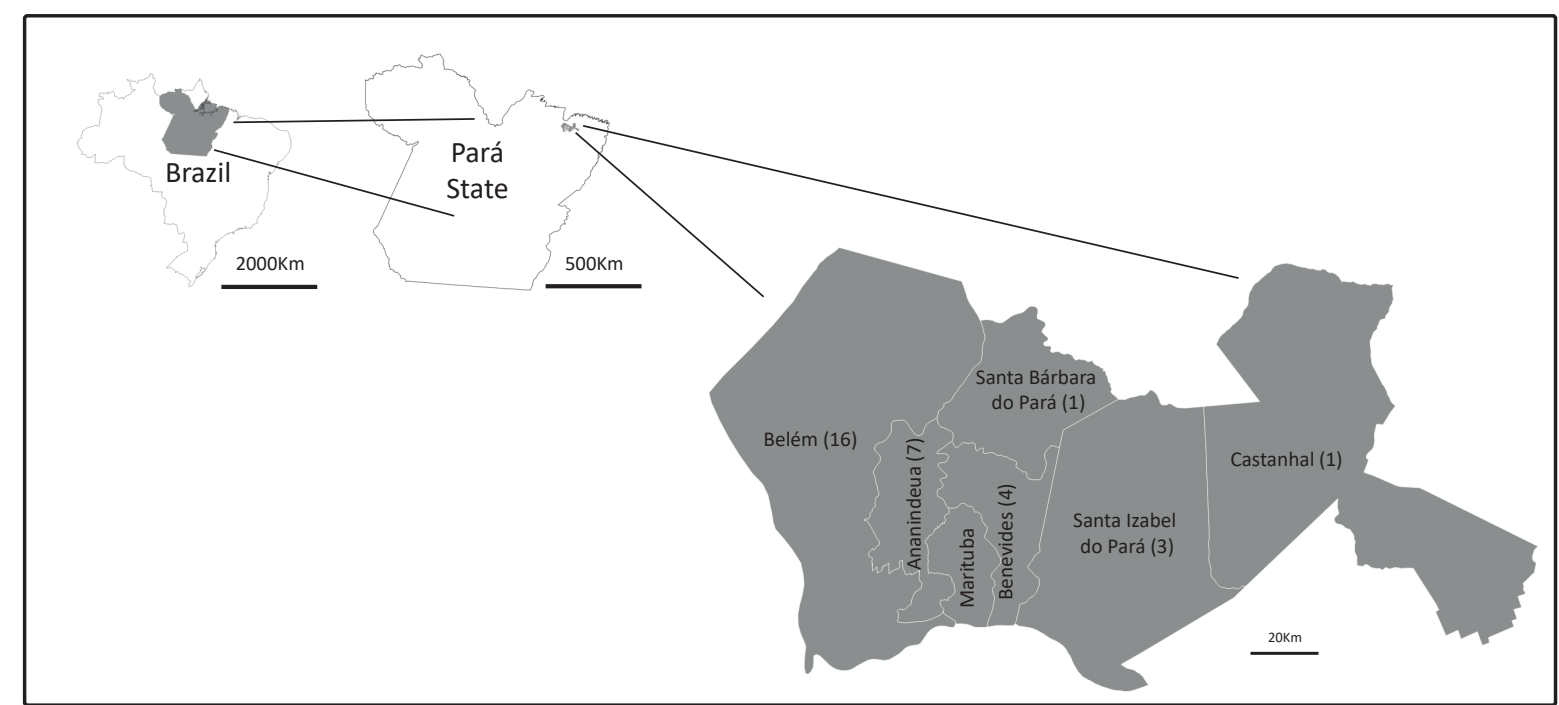

FIGURE 1: Study area; the Belém Metropolitan Region, located in the northeastern Pará State, Brazil. ( ) number of Leishmania isolates (1995-2018) in each municipality. 
DNTP (dATP, dCTP, dGTP, and dTTP) (Quatro G), $0.01 \mu \mathrm{M}$ of each primer (Invitrogen), $2.5 \mathrm{U}$ of Taq DNA polymerase (Invitrogen), and $10 \mu \mathrm{L}$ of extracted DNA $(1 \mathrm{ng} / \mu \mathrm{L})$. The reactions were performed in an Eppendorf(Mastercycler ${ }^{\circledR}$ personal) thermal cycler programmed for an initial denaturation temperature of $94^{\circ} \mathrm{C}$ for $5 \mathrm{~min}$, followed by 40 cycles of $94^{\circ} \mathrm{C}$ for $30 \mathrm{~s}, 55^{\circ} \mathrm{C}$ for $30 \mathrm{~s}$, and $72^{\circ} \mathrm{C}$ for $1 \mathrm{~min}$. The final extension step was maintained for $5 \mathrm{~min}$ at $72^{\circ} \mathrm{C}$. The PCR products were applied to a $1 \%$ agarose gel and stained with Safe Dye (Kasvi) to confirm proper amplification.

A total of $15 \mu \mathrm{L}$ of the PCR product was digested with $10 \mathrm{U}$ of $T s p$ RI (New England Biolabs) $\left(2 \mathrm{~h}\right.$ at $\left.65^{\circ} \mathrm{C}\right)$ or with $2 \mathrm{U}$ of $\mathrm{Hga \textrm {I }}$ (New England Biolabs) endonucleases $\left(2 \mathrm{~h}\right.$ at $\left.37^{\circ} \mathrm{C}\right)$, following the manufacturer's recommendations. Both digestions ( $H g a \mathrm{I}$ and $T s p \mathrm{RI})$ were performed separately for each $15 \mu \mathrm{L}$ of PCR product in a final volume of $20 \mu \mathrm{L}$. The digestion mixtures were individually applied to $3 \%$ agarose gels and stained with Safe dye.

\section{Molecular characterization of Leishmania spp. isolates}

The molecular characterization of Leishmania spp. isolates from patients with ACL in the BMR was based on the RNAPOIILSPCR-RFLP analysis in accordance with previously published studies $^{19,30}$. The following World Health Organization (WHO) Leishmania reference strains preserved in the Ralph Lainson Leishmaniases Lab (Instituto Evandro Chagas) cryobank that had previously been characterized were selected for this analysis: $L$. $(L$.) amazonensis (IFLA/BR/1967/PH8), L. (V.) braziliensis (MHOM/ BR/1975/M2904), L. (V.) guyanensis (MHOM/BR/1975/M4147), L. (V.) naiff (MDAS/BR/1979/M5533), L. (V.) lainsoni (MHOM/ BR/1981/M6426), L. (V.) shawi (MCEB/BR/1984/M8408), and L. (V.) lindenbergi (MHOM/BR/1998/M15732) ${ }^{4}$. However, considering the known genetic diversity of some Leishmania species in the Brazilian Amazon, especially $L$. $(V$.) braziliensis and $L$. $(V$.) guyanensis, another strain of $L$. (V.) braziliensis (MHOM/ BR/1975/M2903), which differs from the above reference strains, was included in this analysis as well as another strain of $L$. $(V$.) guyanensis (MHOM/BR/1997/M16342)(Table 1).

Molecular characterization through RNAPOIILS-PCR-RFLP analysis was based on the reactivity profile of the above WHO
Leishmania reference strains for both $T_{s p} \mathrm{RI}$ and $\mathrm{HgaI}$ endonucleases. Therefore, $T_{s} p$ RI digestion identified three subgroups (SGs): SG1 (no restriction site), corresponding to $L$. (L.) amazonensis; SG2, consisting of $L .(V$.$) guyanensis; and SG3, consisting of L$. $(V$.) braziliensis, L. (V.) naiffi, L. (V.) lainsoni, L. (V.) lindenbergi, and $L$. $(V$.) shawi, while $H g a$ I digestion identified six SGs: SG1 (no restriction site), corresponding to $L$. (L.) amazonensis; SG2, consisting of $L$. (V.) guyanensis, L. (V.) braziliensis (MHOM/ $\mathrm{BR} / 1975 / \mathrm{M} 2904)$, and $L$. ( $V$.) lindenbergi; SG3, consisting of $L$. (V.) naiffi; SG4, consisting of $L$. (V.) lainsoni; SG5, consisting of $L$. (V.) shawi; and SG6, consisting of $L$. $(V$.) braziliensis (MHOM/ BR/1975/M2903). All seven reference strains could be distinguished from one another using the combined analysis of TspRI and $H g a \mathrm{I}$ digestion profiles, with the exception of $L$. (V.) braziliensis (MHOM/ BR/1975/M2904) and L. (V.) lindenbergi (Table 1 and Figure 2).

\section{RESULTS}

\section{Clinical and epidemiological features of $A C L$ in the BMR}

Thirty-two Leishmania spp. isolates were obtained within the historical series analyzed from cutaneous lesions of the LCL clinical form of patients with ACL. Most lesions (75\%; 24/32) were single (ranging from to 1-2) and localized to the arm and/or leg $(81 \%$; 26/32), with reactive Montenegro skin tests ranging at 5-32 mm. The ACL sample comprised patients with a mean age of $32.5 \pm 18.9$ years, predominantly male $(81 \% ; 26 / 32)$, with histories of activities in forested areas (Table 2). All ACL-confirmed cases showed satisfactory treatment responses with no history of relapse for one year post-treatment.

\section{Identification of Leishmania spp. isolates through RNAPOIILS-PCR-RFLP analysis}

The RNAPOIILS-PCR-RFLP analysis of Leishmania spp. DNA products allowed the identification of $14(43.7 \%)$ isolates of $L$. $(V$.) lindenbergi, 11 (34.4\%) of $L$. (V.) lainsoni, four (12.5\%) of $L$. (L.) amazonensis, and three (9.4\%) of $L$. (V.) braziliensis (Table 2). The geographic distributions of the known presumptive contamination sites showed Leishmania species distributions (at the municipality level) as follows: Belém $16(50 \%)[L$. $(V$.) lindenbergi seven, $L$.

TABLE 1: Leishmania spp. WHO reference strains (and closely related others) used for the RNA polymerase II largest subunit (RNAPOIILS) - polymerase chain reaction followed by analysis of restriction fragment length polymorphism (PCR-RFLP) and their respective digestion profiles with TspRI and Hgal endonucleases

\begin{tabular}{ccccc}
\hline Species & Host & WHO code & Locality & $\begin{array}{c}\text { RNAPOILS-PCR-RFLP } \\
\text { profile }\end{array}$ \\
\hline L. (V.) braziliensis & Homo sapiens & MHOM/BR/1975/M2903 & Parauapebas - PA & TspRI SG3 - Hgal SG6 \\
L. (V.) braziliensis & Homo sapiens & MHOM/BR/1975/M2904 & Parauapebas - PA & TspRI SG3 - Hgal SG2* \\
L. (V.) guyanensis & Homo sapiens & MHOM/BR/1975/M4147 & Almeirim - PA & TspRI SG2 - Hgal SG2 \\
L. (V.) guyanensis & Homo sapiens & MHOM/BR/1997/M16342 & Rio Preto da Eva - AM & TspRI SG2 - Hgal SG2 \\
L. (V.) lainsoni & Homo sapiens & MHOM/BR/1981/M6426 & Benevides - PA & TspRI SG3 - Hgal SG4 \\
L. (V.) naiffi & Dasypus novemcintus & MDAS/BR/1979/M5533 & Almeirim - PA & TspRI SG3 - Hgal SG3 \\
L. (V.) shawi & Sapajus apella & MCEB/BR/1984/M8408 & Parauapebas - PA & TspRI SG3 - Hgal SG5 \\
L. (V.) lindenbergi & Homo sapiens & MHOM/BR/1996/M15729 & Belém - PA & TspRI SG3 - Hgal SG2* \\
L. (L.) amazonensis & Bichromomyia flaviscutellata & IFLA/BR/1967/PH8 & Belém - PA & TspRI SG1- Hgal SG1 \\
\hline
\end{tabular}

L.: Leishmania; V.: Viannia; WHO: World Health Organization; PA: Pará State, Brazil; AM: Amazonas State, Brazil. *These different Leishmania species present the same RNAPOIILS-PCR-RFLP profile, requiring additional methods for unambiguous characterization. 


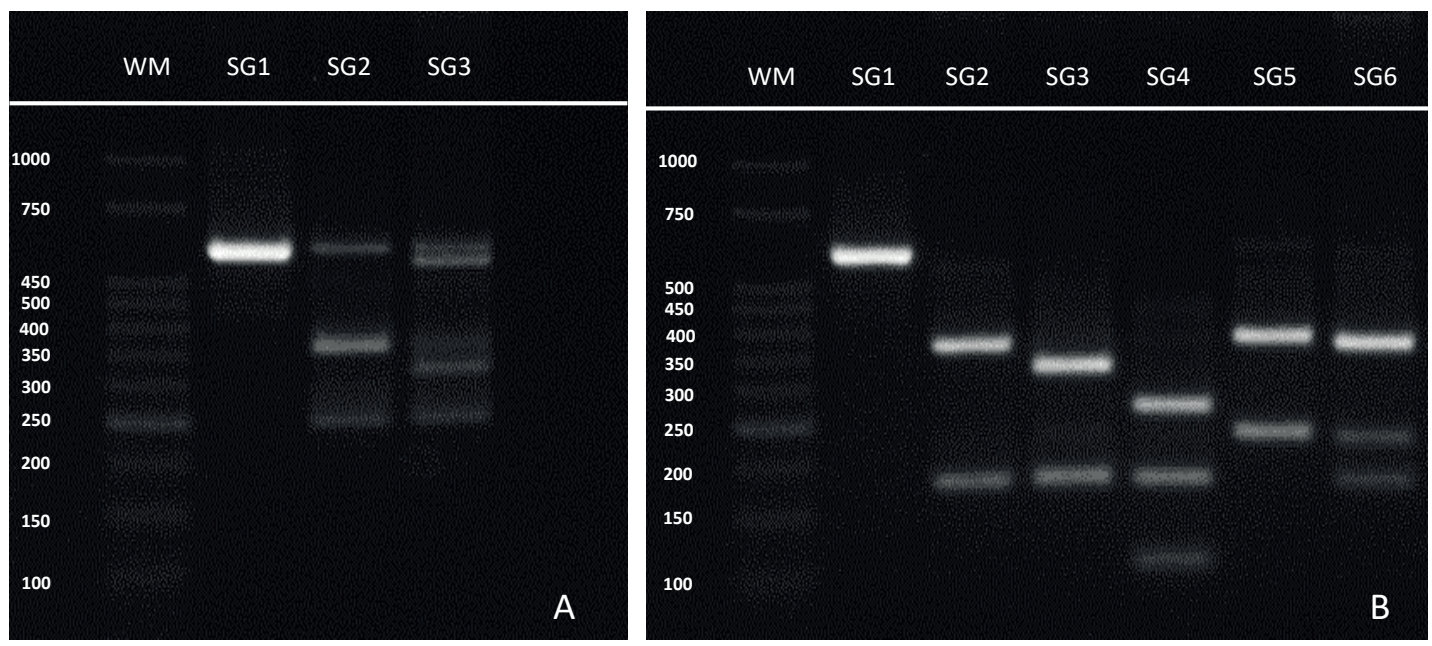

FIGURE 2: RNA polymerase II largest subunit (RNAPOIILS)-polymerase chain reaction followed by analysis of restriction fragment length polymorphism (PCR-RFLP) of Leishmania spp. digestion profiles (TspRI and Hgal endonucleases). (A): TspRI digestion subgroups (SGs): WM, molecular weight marker (in base pairs) (1 kb-Ludwig Biotec); SG1 (no restriction site), corresponding to L. (L.) amazonensis; SG2, consisting of L. (V.) guyanensis; and SG3, consisting of L. (V.) braziliensis, L. (V.) naiffi, L. (V.) lainsoni, L. (V.) lindenbergi, and L. (V.) shawi. (B): Hgal digestion SGs: SG1 (no restriction site), corresponding to L. (L.) amazonensis; SG2, consisting of $L$. (V.) guyanensis, L. (V.) braziliensis (MHOM/BR/1975/M2904), and L. (V.) lindenbergi; SG3, consisting of $L$. (V.) naiffi; SG4, consisting of L. (V.) lainsoni; SG5, consisting of L. (V.) braziliensis (MHOM/BR/1975/M2903); and SG6, consisting of $L$. (V.) shawi.

TABLE 2: Leishmania spp. isolates from patients with American cutaneous leishmaniasis from the Belém Metropolitan Region (1995-2018), characterized by RNA polymerase II largest subunit (RNAPOIILS)-polymerase chain reaction followed by analysis of restriction fragment length polymorphism (PCR-RFLP).

\begin{tabular}{|c|c|c|c|c|c|c|c|c|}
\hline Mnemonic & Infection site & Sex & Age & Lesions & MST (mm) & WHO code & RNAPOIILS-PCR-RFLP profile & Leishmania species \\
\hline SNFS & Ananindeua & M & 22 & 2 (leg) & n.a. & MHOM/BR/1995/M15265 & TspRI SG3 - Hgal SG4 & L. (V.) lainsoni \\
\hline EBR & Ananindeua & $\mathrm{F}$ & 17 & 1 (leg) & n.a. & MHOM/BR/1995/M15418 & TspRI SG1- Hgal SG1 & L. (V.) amazonensis \\
\hline LSM & Ananindeua & M & 21 & 1 (leg) & n.a. & MHOM/BR/1996/M15720 & TspRI SG1- Hgal SG1 & L. (V.) amazonensis \\
\hline JMS & Belém & M & 20 & 1 (arm) & $17 \times 17$ & MHOM/BR/1996/M15279 & TspRI SG3 - Hgal SG2 & L. (V.) lindenbergi \\
\hline OPR & Benevides & M & 19 & 2 (hand) & $32 \times 32$ & MHOM/BR/1996/M15732 & TspRI SG3 - Hgal SG2 & L. (V.) lindenbergi \\
\hline WNSS & Belém & M & 19 & 1 (arm) & n.a. & MHOM/BR/1996/M15740 & TspRI SG3 - Hgal SG2 & L. (V.) lindenbergi \\
\hline AOM & Belém & M & 20 & 1 (leg) & $10 \times 10$ & MHOM/BR/1996/M15819 & TspRI SG3 - Hgal SG2 & L. (V.) lindenbergi \\
\hline PCB & Belém & M & 43 & 1 (back) & n.a. & MHOM/BR/1999/M18048 & TspRI SG3 - Hgal SG4 & L. (V.) Iainsoni \\
\hline LFB & Belém & $\mathrm{F}$ & 38 & 1 (arm) & $7 \times 9$ & MHOM/BR/1999/M18054 & TspRI SG3 - Hgal SG4 & L. (V.) lainsoni \\
\hline PGP & Belém & $M$ & 50 & 2 (leg) & $12 \times 12$ & MHOM/BR/2000/M18820 & TspRI SG3 - Hgal SG2 & L. (V.) lindenbergi \\
\hline MDSA & Belém & M & 20 & 1 (arm) & n.a. & MHOM/BR/2000/M19418 & TspRI SG3 - Hgal SG2 & L. (V.) lindenbergi \\
\hline WGH & Benevides & M & 41 & 2 (leg) & $8 \times 8$ & MHOM/BR/2000/M19477 & TspRI SG3 - Hgal SG6 & L. (V.) braziliensis \\
\hline VLS & Castanhal & $M$ & 21 & 1 (arm) & n.a. & MHOM/BR/2000/M19480 & TspRI SG3 - Hgal SG6 & L. (V.) braziliensis \\
\hline PAN & Santa Barbara & M & 45 & 1 (arm) & $9 \times 9$ & MHOM/BR/2001/M20321 & TspRI SG3 - Hgal SG2 & L. (V.) lindenbergi \\
\hline JBS & Santa Izabel & M & 32 & 1 (nose) & n.a. & MHOM/BR/2002/M21484 & TspRI SG3 - Hgal SG4 & L. (V.) lainsoni \\
\hline LSB & Ananindeua & M & 16 & 1 (leg) & $14 \times 14$ & MHOM/BR/2003/M22090 & TspRI SG3 - Hgal SG4 & L. (V.) lainsoni \\
\hline FDS & Belém & $M$ & 30 & 2 (leg) & $10 \times 10$ & MHOM/BR/2009/M26488 & TspRI SG3 - Hgal SG2 & L. (V.) lindenbergi \\
\hline EAO & Belém & M & 44 & 1 (head) & $10 \times 10$ & MHOM/BR/2011/M28690 & TspRI SG3 - Hgal SG4 & L. (V.) lainsoni \\
\hline EMO & Belém & M & 44 & 1 (leg) & $10 \times 10$ & MHOM/BR/2012/M29080 & TspRI SG3 - Hgal SG4 & L. (V.) lainsoni \\
\hline DGA & Benevides & M & 20 & 2 (arm) & $10 \times 10$ & MHOM/BR/2012/M29084 & TspRI SG3 - Hgal SG2 & L. (V.) lindenbergi \\
\hline ELG & Belém & M & 25 & 1 (face) & $14 \times 14$ & MHOM/BR/2013/M29809 & TspRI SG3 - Hgal SG4 & L. (V.) lainsoni \\
\hline SGA & Benevides & $\mathrm{F}$ & 62 & 1 (leg) & $8 \times 8$ & MHOM/BR/2013/M29986 & TspRI SG3 - Hgal SG2 & L. (V.) lindenbergi \\
\hline OMP & Ananindeua & M & 33 & 1 (leg) & $13 \times 14$ & MHOM/BR/2013/M30178 & TspRI SG3 - Hgal SG2 & L. (V.) lindenbergi \\
\hline $\mathrm{CPL}$ & Belém & M & 7 & 2 (face/arm) & $5 \times 5$ & MHOM/BR/2014/M30464 & TspRI SG3 - Hgal SG4 & L. (V.) lainsoni \\
\hline HMM & Belém & $\mathrm{F}$ & 19 & 1 (leg) & n.a. & MHOM/BR/2014/M30800 & TspRI SG1- Hgal SG1 & L. (V.) amazonensis \\
\hline THS & Belém & $\mathrm{F}$ & 79 & 1 (arm) & $15 \times 15$ & MHOM/BR/2014/M30921 & TspRI SG3 - Hgal SG2 & L. (V.) lindenbergi \\
\hline WNSC & Belém & M & 7 & 2 (face) & $30 \times 30$ & MHOM/BR/2015/M31232 & TspRI SG3 - Hgal SG4 & L. (V.) Iainsoni \\
\hline RFF & Santa Izabel & M & 89 & 1 (face) & n.a. & MHOM/BR/2015/M31234 & TspRI SG3 - Hgal SG4 & L. (V.) lainsoni \\
\hline OSP & Santa Izabel & M & 44 & 1 (arm) & $5 \times 5$ & MHOM/BR/2015/M31462 & TspRI SG1- Hgal SG1 & L. (V.) amazonensis \\
\hline PLBS & Ananindeua & $\mathrm{F}$ & 16 & 1 (leg) & $9 \times 9$ & MHOM/BR/2015/M31702 & TspRI SG3 - Hgal SG2 & L. (V.) lindenbergi \\
\hline FEGA & Belém & M & 41 & 1 (arm) & $12 \times 12$ & MHOM/BR/2016/M31799 & TspRI SG3 - Hgal SG6 & L. (V.) braziliensis \\
\hline WFS & Ananindeua & $\mathrm{M}$ & 36 & 1 (leg) & $10 \times 10$ & MHOM/BR/2018/M32884 & TspRI SG3 - Hgal SG2 & L. (V.) lindenbergi \\
\hline
\end{tabular}

WHO: World Health Organization; MST: Montenegro skin test; n.a.: not available. 


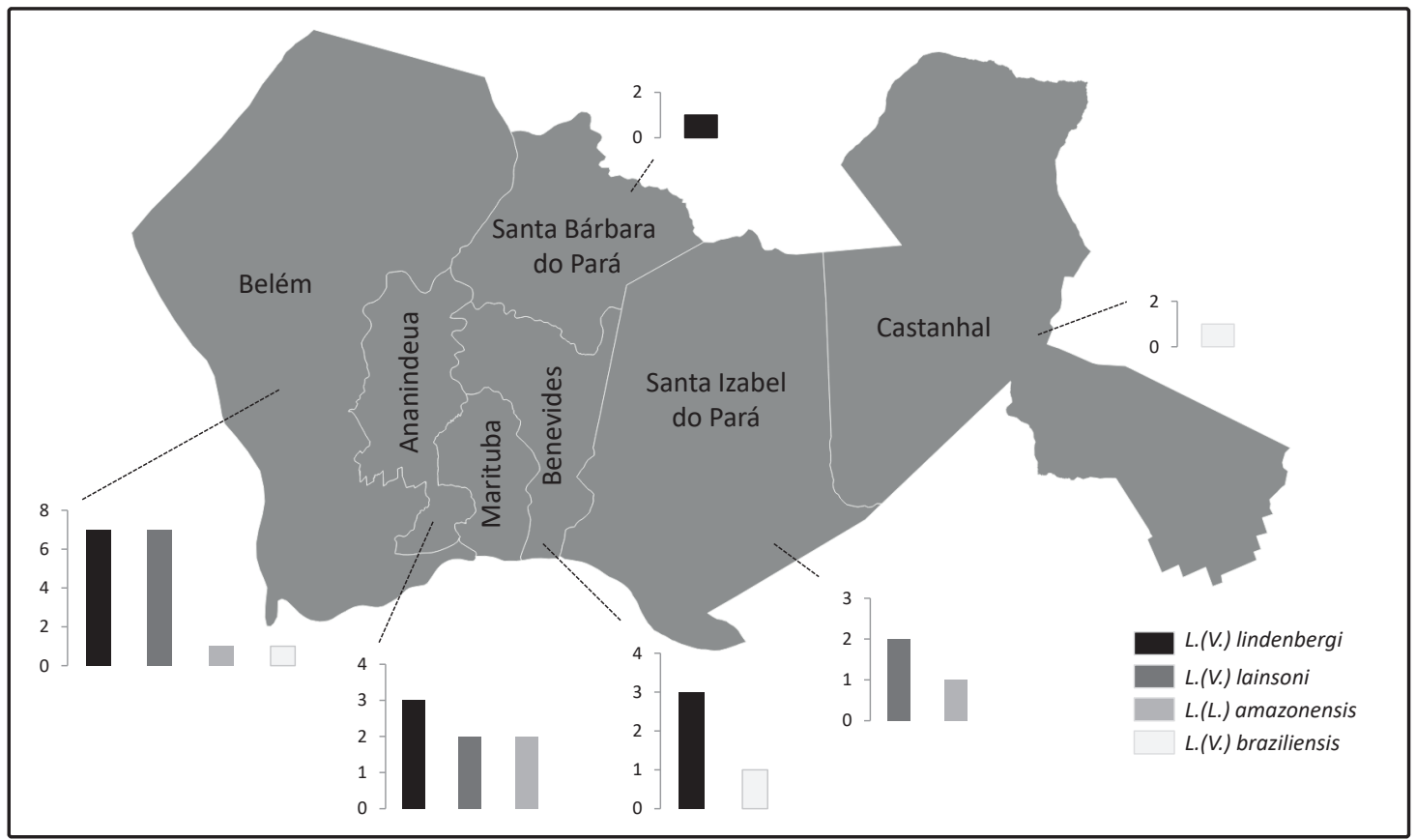

FIGURE 3: Distribution of Leishmania species in the municipalities of the Belém Metropolitan Region.

(V.) lainsoni seven, L. (L.) amazonensis one, and $L$. (V.) braziliensis one], Ananindeua seven (21.8\%) $[L .(V$.$) lindenbergi three, L .(V)$. lainsoni two, and $L$. (L.) amazonensis two], Benevides four (12.5\%) $[L .(V$.$) lindenbergi three and L .(V$.$) braziliensis one], Santa Izabel$ do Pará three (9.5\%) [L. (V.) lainsoni two and L. (L.) amazonensis one], Santa Bárbara do Pará one (3.1\%) [L. (V.) lindenbergi], and Castanhal one (3.1\%) [L. (V.) braziliensis] (Figure 3).

The combined analysis of the TspRI and HgaI digestion profiles of the isolates of $L$. (V.) lindenbergi, $L$. $(V$.) lainsoni, and $L$. (L.) amazonensis presented the same RNAPOIILS-PCR-RFLP profiles as their respective WHO Leishmania reference strains. However, considering that the two $L$. $(V$.) braziliensis WHO Leishmania reference strains (MHOM/BR/1975/M2904 and MHOM/BR/1975/M2903) showed two distinct patterns upon $\mathrm{HgaI}$ digestion, all $L$. $(V$.) lindenbergi isolates could be distinguished from $L$. (V.) braziliensis MHOM/BR/1975/M2903, but not from the MHOM/BR/1975/M2904 strain. Likewise, all three isolates of L. (V.) braziliensis were identified based on the MHOM/BR/1975/ M2903 RNAPOIILS-PCR-RFLP profile (Table 2). To clarify this ambiguous reactivity (TspRI and $H g a \mathrm{I}$ ) between $L$. (V.) lindenbergi and $L$. (V.) braziliensis (MHOM/BR/1975/M2904), the biological behavior of experimentally infected hamsters was checked for all 14 isolates of $L$. $(V$.) lindenbergi after the parasite was isolated, which revealed that none of these isolates could produce apparent ulcerated cutaneous lesion at the "hamster" inoculation site (hindfoot), suggesting a typical behavior of $L$. $(V$.) lindenbergi infection and not of $L$. $(V$.) braziliensis infection.

\section{DIscussion}

DNA-based methods have been extensively used since the 1980s for the characterization of Leishmania spp. However, these methods are currently restricted to referral hospitals and research centers with well-equipped laboratories. Currently available techniques include direct sequencing of a PCR product, use of species-specific restriction sites via PCR-RFLP, PCR fingerprinting, random amplified polymorphic DNA, or high-resolution melting. Of these, only PCR-RFLP and sequence analysis coupled with the appropriate target in the genome are suitable for the discrimination of all Leishmania species. In many cases, a combination of different markers must be applied to achieve a definitive taxonomic resolution ${ }^{13,14}$.

The characterization of Leishmania spp. has traditionally been performed in the Ralph Lainson Leishmaniases Lab (since the 1970s) using a combined methodology that takes into consideration the parasite's behavior within experimentally infected invertebrate (phebotomines) and vertebrate (hamsters) hosts in the Dfico B ${ }^{45}$ culture medium, but has been improved with the "gold standard" MLEE and IFAT-Mabs analyses ${ }^{8,26-29}$. We extended the applicability of a simple and direct molecular tool that was originally proposed (and recently revised) for French Guiana ${ }^{19,30}$, and used it to identify (to date) the coexisting human-pathogenic dermotropic Leishmania species in the BMR. This methodology has already been used to identify Leishmania isolates from patients with ACL in our immunopathology research laboratory ${ }^{31}$ as well as from phlebotomines ${ }^{24,29}$. The sensitivity of RNAPOIILS-PCR-RFLP was $100 \%$, as expected for isolates. High specificity was also presumed, since the RNAPOIILS-PCR-RFLP for Leishmania profiles is distinct from that for other microorganisms. For non-isolated samples, future steps will include the analysis of Giemsa-stained lesion imprint slides, which have a presumed sensitivity of approximately $90 \%{ }^{19}$, to improve sensitivity for Leishmania DNA detection. These samples can be preliminarily screened with markers targeting kDNA. 
The RNAPOIILS-PCR-RFLP profiles of $L$. (L.) amazonensis, L. (V.) braziliensis, L. (V.) guyanensis, $L$. (V.) lainsoni, and $L$. (V.) naiffi have been published previously ${ }^{19}$, although, to our knowledge, this is the first analysis of $L$. (V.) shawi and $L$. (V.) lindenbergi, thus, providing an extension of the species-specific distinction power of this methodology. The analysis of some Leishmania species with high polymorphic genetic potential can be challenging. In this sense, $L$. (V.) guyanensis from eastern and western Amazonia are antigenically distinct when analyzed by IFAT-Mabs ${ }^{32}$, and a degree of intra-specific heterogeneity has been observed in distinct populations from French Guiana using a small subunit and internal transcribed spacer 1 in the rRNAgenes-PCR-RFLP analysis ${ }^{33}$. Despite these observations, no intraspecific variation was observed in the RNAPOIILS-PCR-RFLP analysis.

Additionally, in the present study, $L$. (V.) braziliensis, a widely distributed and potentially polymorphic species ${ }^{34,35}$, revealed different RNAPOIILS-PCR-RFLP profiles of two samples from the same geographical area in the "Serra dos Carajás" southeastern region of the Pará State (MHOM/BR/1975/2904 [Serra Norte N1] and MHOM/BR/1975/M2903 [Serra Norte H7]), with the profile of the former being indistinguishable from that of $L$. ( V.) lindenbergi. This indicates a limitation of PCR-RFLP analysis when referring to an ecological region with a considerable genetic diversity of Leishmania parasites. This was also observed in another study in the Brazilian Amazon that failed to distinguish $L$. (V.) lindenbergi from $L$. (V.) guyanensis through hsp 70 PCR-RFLP (with HaeIII digestion). In this scenario, the authors utilized 6-phosphogluconate dehydrogenase, glucose-6-phosphate dehydrogenase MLEE, the analysis of partial sequences of $h s p 70$, isocitrate dehydrogenase, and mannose phosphate isomerase genes for final identification ${ }^{36}$. However, in the present study, the biological behavior (experimental infection in "hamster") of all 14 isolates of suspected $L$. $(V$.) lindenbergi when the parasite was isolated revealed that none of these isolates could produce conspicuous ulcerated cutaneous lesions at the inoculation site (footpad) of laboratory animals, thus, confirming the typical biological behavior of $L$. (V.) lindenbergi infection. Another point that contradicts this high number (14) of isolates being $L$. ( V.) braziliensis is that in over 40 years of research at the BMR, we have never seen a case of mucocutaneous leishmaniasis originating from this region (Silveira, personal communication).

The present study represents the first systematic study to examine, mainly through molecular methods, the repertoire of Leishmania species occurring in this region, and to our knowledge, revealing for the first time that $L$. $(V$.) lindenbergi (43.7\%) and $L$. $(V$ ) lainsoni $(34.4 \%)$ are the main ACL agents in the BMR, followed by $L$. (L.) amazonensis $(12.5 \%)$ and $L$. (V.) braziliensis $(9.4 \%)$. From an eco-epidemiological point of view, it is interesting to note that these Leishmania spp. enzootics in the BMR remain established in residual forest fragments with ecological conditions, such as the presence of phlebotomine potential vectors ${ }^{37,38}$, which favor Leishmania life cycles. Clinical and socioeconomic data show ACL in the BMR as a predominantly accidental disease with an occupational/eco-touristic character, since it has mainly been associated with middle-aged men exposed to peri-urban forest environments. Occasional ACL cases of patients with no history of forest exposure (such as elderly housewives) have drawn attention to its potential for intra/peridomiciliary transmission.
The adaptation of $L$. ( $V$.) lindenbergi and $L$. (V.) lainsoni enzootic cycles to the current ACL ecological scenario in the BMR is interesting. These species are together responsible for the majority $(81.3 \%)$ of all ACL cases examined in this study. While $L$. (V.) lainsoni has already been found in the Brazilian Amazon States of Pará, Amapá ${ }^{39}$, Rondônia ${ }^{40}$, and Acre ${ }^{41}$ as well as in other South American countries/territories, such as Peru ${ }^{42}$, Bolivia $^{43}$, Colombia ${ }^{44}$, Suriname ${ }^{45}$, French Guiana ${ }^{19}$, and Ecuador ${ }^{46}$, $L$. (V.) lindenbergi has, until recently, not been recorded outside its type-locality, the BMR. The first report of $L$. (V.) lindenbergi causing ACL occurred in Rondônia State, Brazilian western Amazon region ${ }^{36}$. Underreporting of ACL due to this parasite is possible, since some methodologies currently employed for Leishmania identification may not distinguish this species from others. Therefore, increasing efforts to develop novel techniques for species identification in other Amazonian regions may expand our knowledge on the geographical range of $L$. (V.) lindenbergi.

In this study, we record the first three cases of ACL due to L. (V.) braziliensis in the BMR, with strains from the municipalities of Belém, Benevides, and Castanhal being compatible with the MHOM/BR/1975/M2903 RNAPOIILS-PCR-RFLP profile, thus, distinct from that of $L$. $(V$.) lindenbergi. The ecological scenario of $L$. (V.) braziliensis is not yet well understood in this region, as the well-known vectors Psychodopygus wellcomei/Psychodopygus complexus have not yet been recorded in surveyed forest fragments in the BMR ${ }^{11,37,38}$. Alternative transmission (most likely by other 'psychodopygians'), thus, cannot be ruled out. Psychodopygus davisi, for instance, is a very active human-biting phlebotomine species present in the BMR that was found to be infected with L. (V.) braziliensis in the southern Pará State ${ }^{47}$.

In summary, the results of this study provide a better understanding of the ACL epidemiological scenario in the BMR. Strong ecological transformations have occurred in this region over the years, although these changes do not appear to limit the enzootic cycles of the Leishmania species already identified here.

\section{ETHICAL STANDARDS}

Procedures involving humans were submitted and approved by the Comitê de Ética em Pesquisa - CEP (Ethics in Research Committee), under protocol CAAE 95080418.0000.0019. Procedures involving access to stored material of non-human vertebrates were submitted and approved by the Comissão de Ética no Uso de Animais - CEUA (Ethics in Animal Use Commission), under protocol CEUA/IEC/SVS/MS n.42/2018.

\section{ACKNOWLEDGMENTS}

We wish to thank to Ghislaine Prévot and Marine Ginouvès (Département de Médecine, Université de Guyane, Cayenne, French Guiana) for their advice on the RNAPOIILS-PCR-RFLP training given to one of us (TVS). We are also grateful to the Ralph Lainson's Leishmaniasis Lab team, in particular to Ana Camila Oliveira Alves and Ádria de Oliveira Castro for their technical support given to the molecular analysis; to Domingas Ribeiro Everdosa and João Batista Palheta da Luz for their technical support with the laboratory diagnosis of the patients, and to Raimundo Nonato Barbosa Pires for his technical support and curatorial care with the Leishmania cryobank. 


\section{FINANCIAL SUPPORT}

This research was financially supported by the Instituto Evandro Chagas (Brazilian Ministry of Health) and by the Núcleo de Medicina Tropical (Universidade Federal do Pará, Brasil). LPG received scholarship from the Conselho Nacional de Desenvolvimento Científico e Tecnológico (editals no.1/2017PIBIC/IEC/CNPq 2017-2018 and no.1/2016-PIBIC/IEC/CNPq 2016-2017).

\section{AUTHOR'S CONTRIBUTION}

LPG: Original draft, Data collection, Conduction of experiments, Data analysis, Final draft; TVS: Study design/conception, Original draft, Data analysis, Final draft; MBC: Data collection, Data analysis, Final draft; LVLR: Data analysis, Final draft; EAYI: Data analysis, Final draft; FTS: Study design/conception, Data collection, Data analysis, Final draft; PKSR: Study design/conception, Original draft, Data collection, Conduction of experiments, Data analysis, Final draft.

\section{CONFLICT OF INTEREST}

The authors declare that there is no conflict of interest.

\section{REFERENCES}

1. Espinosa OA, Serrano MG, Camargo EP, Teixeira MMG, Shaw JJ. An appraisal of the taxonomy and nomenclature of trypanosomatids presently classified as Leishmania and Endotrypanum. Parasitology. 2018;145(4):430-42.

2. Lainson R, Shaw JJ. New World Leishmaniasis, in Topley \& Wilson's Microbiology and Microbial Infections. John Wiley \& Sons. 2010.

3. Silveira FT, Lainson R, Muller SFR, De Souza AAA, Corbett CEP. Leishmaniose tegumentar Americana, in Medicina Tropical e Infectologia na Amazônia, Leão RNQ , Editor. Belém, Brazil: Samaúma, 2013. pp.1203-44.

4. Jennings YL, de Souza AAA, Ishikawa EA, Shaw J, Lainson R, Silveira F. Phenotypic characterization of Leishmania spp. causing cutaneous leishmaniasis in the lower Amazon region, western Pará state, Brazil, reveals a putative hybrid parasite, Leishmania (Viannia) guyanensis $\times$ Leishmania (Viannia) shawi shawi. Parasite. 2014;21(39).

5. Lainson R, Shaw JJ, Silveira FT, Souza AAA, Braga RR, Ishikawa EAY. The dermal leishmaniases of Brazil, with special reference to the eco-epidemiology of the disease in Amazonia. Mem Inst Oswaldo Cruz. 1994;89(3):435-43.

6. Lainson R, Shaw JJ. Evaluation, classification and geographical distribution, in The leishmaniases, biology and medicine, Peters W, Killick-Kendrick R, Editors. London: Academic Press. 1987; p.1-120.

7. Lainson R. The American leishmaniases: some observations on their ecology and epidemiology. Trans R Soc Trop Med Hyg. 1983;77(5):569-96.

8. Lainson R. The Neotropical Leishmania species: a brief historical review of their discovery, ecology and taxonomy. Rev Pan-Amaz Saude. 2010;1(2):13-32.

9. Lainson R, Shaw JJ. Leishmaniasis of the New World: taxonomic problems. Br Med Bull. 1972;28(1):44-8.

10. Silveira FT, Shaw JJ, Braga RR, Ishikawa E. Dermal leishmaniasis in the Amazon region of Brazil: Leishmania (Viannia) lainsoni sp. n., a new parasite from the State of Pará. Mem Inst Oswaldo Cruz. 1987;82(2):289-91
11. Silveira FT, Ishikawa EAI, de Souza AAA, Lainson R. An outbreak of cutaneous leishmaniasis among soldiers in Belém, Pará State, Brazil caused by Leishmania (Viannia) lindenbergi n. sp., a new leishmanial parasite of man in the Amazon region. Parasite. 2002;9(1):43-50.

12. World Health Organization. Control of the Leishmaniasis: Report of a meeting of the WHO Expert Committee on the Control of Leishmaniasis, Geneva, (WHO technical report series ; no. 949). 2010; 186pp.

13. Van der Auwera G, Dujardin J-C. Species typing in dermal leishmaniasis. Clin Microbiol Rev. 2015; 28(2):265-94.

14. Akhoundi M, Downing T, Votýpka J, Kuhls K, Lukeš J, Cannet A, et al. Leishmania infections: Molecular targets and diagnosis. Mol Aspects Med. 2017;57:1-29.

15. Garcia FCB, Santos SSR, Chociay MF, Medeiros ACR, Roselino AMF. Subsidiary methods for the diagnosis of American tegumentar leishmaniasis (ATL): comparison of sequencing of DNA and PCRRFLP for identification of Leishmania species in skin samples. Anais Brasileiros de Dermatologia. 2005;80(suppl.3):S339-44.

16. Graça GC, Volpini AC, Romero GA, Oliveira Neto MP, Hueb M, Porrozzi R, et al. Development and validation of PCR-based assays for diagnosis of American cutaneous leishmaniasis and identification of the parasite species. Mem Inst Oswaldo Cruz. 2012;107(5):664-74.

17. Monroy-Ostria A, Nasereddin A, Monteon VM, Guzmán-Bracho C, Jaffe CL. ITS1 PCR-RFLP Diagnosis and characterization of Leishmania in clinical samples and strains from cses of human cutaneous leishmaniasis in states of the Mexican Southeast. Interdiscip Perspect Infect Dis. 2014;2014:1-6.

18. Montalvo AM, Fraga J, Maes I, Dujardin JC, Van der Auwera G. Three new sensitive and specific heat-shock protein 70 PCRs for global Leishmania species identification. Eur J Clin Microbiol Infect Dis. 2012;31(7):1453-61.

19. Simon S, Veron V, Carme B. Leishmania spp. identification by polymerase chain reaction-restriction fragment length polymorphism analysis and its applications in French Guiana. Diagn Microbiol Infect Dis. 2010;66(2):175-80.

20. Croan DG, Morrison DA, Ellis JT. Evolution of the genus Leishmania revealed by comparison of DNA and RNA polymerase gene sequences. Mol Biochem Parasitol. 1997;89(2):149-59.

21. IBGE - Estimativas da população residente para os municípios e para as unidades da federação brasileiros com data de referência em $1^{\circ}$ de julho de 2017. 2017,11p.

22. Leão N, Alencar C, Veríssimo A. Belém Sustentável 2007. Belém, Brazil: Instituto do Homem e Meio Ambiente da Amazônia, 2008.

23. Silveira FT, Lainson R, Corbett CEP. Further observations on clinical, histopathological, and immunological features of borderline disseminated cutaneous leishmaniasis caused by Leishmania (Leishmania) amazonensis. Mem Inst Oswaldo Cruz. 2005;100(5):525-34.

24. Vasconcelos dos Santos T, Prévot G, Ginouvès M, Duarte R, Silveira FT, Póvoa MM, et al. Ecological aspects of Phlebotomines (Diptera: Psychodidae) and the transmission of American cutaneous leishmaniasis agents in an Amazonian/ Guianan bordering area. Parasit Vectors. 2018;11(1):612.

25. Brasil - Ministry of Health. Secretary of Surveillance in Health. Department of Surveillance in Transmissible Diseases. 2017. Guide to surveillance of tegumentary leishmaniasis [in Portuguese]. 2nd ed. Brasília: Ministério da Saúde press; 189p.

26. Miles MA, Lainson R, Shaw JJ, Póvoa MM, de Souza AAA. Leishmaniasis in Brazil: XV. Biochemical distinction of Leishmania mexicana amazonensis, Leishmania braziliensis braziliensis and Leishmania braziliensis guyanensis - aetiological agents of cutaneous 
leishmaniasis in the Amazon Basin of Brazil. Trans R Soc Trop Med Hyg. 1981;75(4):524-9.

27. Miles MA, Póvoa MM, de Souza AAA, Lainson R, Shaw JJ. Some methods for the enzymic characterization of Latin-American Leishmania, with particular reference to Leishmania mexicana amazonensis and subspecies of Leishmania hertigi. Trans R Soc Trop Med Hyg. 1980;74(2):243-52.

28. Shaw JJ, Ishikawa EA, Lainson R. A rapid and sensitive method for the identification of Leishmania with monoclonal antibodies using fluorescein-labelled avidin. Trans R Soc Trop Med Hyg. 1989;83(6):7834.

29. Souza AAA, Barata IR, Silva MGS, Lima JAN, Jennings YLL, Ishikawa EAY, et al. Natural Leishmania (Viannia) infections of phlebotomines (Diptera: Psychodidae) indicate classical and alternative transmission cycles of American cutaneous leishmaniasis in the Guiana Shield, Brazil. Parasite. 2017;24:13.

30. SimonS,NacherM,CarmeB,BasurkoC,RogerA,AdenisA, etal.Cutaneous leishmaniasis in French Guiana: revising epidemiology with PCRRFLP. Trop Med Health. 2017;45:5.

31. Campos MB, Lima LVdR, de Lima ACS, Vasconcelos dos Santos T, Ramos PKS, Gomes CMC, et al. Toll-like receptors 2, 4, and 9 expressions over the entire clinical and immunopathological spectrum of American cutaneous leishmaniasis due to Leishmania (V.) braziliensis and Leishmania (L.) amazonensis. PLoS ONE. 2018;13(3):e0194383.

32. Romero GAS, Ishikawa E, Cupolillo E, Toaldo CB, Guerra MVF, Paes MG, et al. Identification of antigenically distinct populations of Leishmania (Viannia) guyanensis from Manaus, Brazil, using monoclonal antibodies. Acta Trop. 2002;82(01):25-9.

33. Rotureau B, Ravel C, Nacher M, Couppie P, Curtet I, Dedet JP, et al. Molecular Epidemiology of Leishmania (Viannia) guyanensis. in French Guiana. J Clin Microbiol. 2006;44(2):468-73.

34. Cupolillo E, Brahim LR, Toaldo CB, de Oliveira-Neto MP, de Brito ME, Falqueto A, et al. Genetic polymorphism and molecular epidemiology of Leishmania (Viannia) braziliensis from different hosts and geographic areas in Brazil. J Clin Microbiol. 2003;41(7):3126-32.

35. Ishikawa EA, Silveira FT, Magalhães AL, Guerra Júnior RB, Melo MN, Gomes R, et al. Genetic variation in populations of Leishmania species in Brazil. Trans R Soc Trop Med Hyg. 2002;96(Suppl 1):S111-21.

36. Cantanhêde LM, Mattos CB, de Souza Ronconi C, Filgueira CPB, da Silva Júnior CF, Limeira C, et al. First report of Leishmania (Viannia) lindenbergi causing tegumentary leishmaniasis in the Brazilian western Amazon region. Parasite. 2019;26:30.
37. Ferreira JVS, Vasconcelos dos Santos T, Santos EM, Gorayeb IS. Phlebotomine sand flies (Diptera: Psychodidae) in forest fragments of Belém metropolitan area, Pará State, Brazil, with considerations on vectors of American cutaneous leishmaniasis agents. Rev Pan-Amaz Saude. 2014;5(2):29-35.

38. Sánchez-Uzcátegui YdV, Vasconcelos dos Santos T, Sulveira FT, Ramos PKS, Santos EJM, Póvoa MM. Phlebotomines (Diptera: Psychodidae) from a Urban Park of Belém, Pará State, Northern Brazil and Potential Implications in the Transmission of American Cutaneous Leishmaniasis. Journal of Medical Entomology. 2020;57(1): 281-8.

39. Silveira FT, Souza AA, Lainson R, Shaw JJ, Braga RR, Ishikawa EAY. Cutaneous leishmaniasis in the Amazon region: natural infection of the sandfly Lutzomyia ubiquitalis (Psychodidae: Phlebotominae) by Leishmania (Viannia) lainsoni in Pará State, Brazil. Mem Inst Oswaldo Cruz. 1991;86(1):127-30.

40. Cantanhêde LM, da Silva Júnior CF, Ito MM, Felipin KP, Nicolete R, Salcedo JMV, et al. Further Evidence of an association between the presence of Leishmania RNA Virus 1 and the mucosal manifestations in tegumentary leishmaniasis Patients. PLoS Negl Trop Dis. 2015;9(9):e0004079.

41. Tojal da Silva AC, Cupolillo E, Volpini AC, Almeida R, Romero GA. Species diversity causing human cutaneous leishmaniasis in Rio Branco, state of Acre, Brazil. Trop Med Int Health. 2006;11(9):1388-98.

42. Lucas CM, Franke ED, Cachay MI, Tejada A, Carrizales D, Kreutzer RD. Leishmania (Viannia) lainsoni: first isolation in Peru. Am J Trop Med Hyg. 1994;51(5):533-7.

43. Martinez E, Le Pont F, Mollinedo S, Cupolillo E. A first case of cutaneous leishmaniasis due to Leishmania (Viannia) lainsoni in Bolivia. Trans R Soc Trop Med Hyg. 2001;95(4):375-7.

44. Patino LH, Mendez C, Rodriguez O, Romero Y, Velandia D, Alvarado $\mathrm{M}$, et al. Spatial distribution, Leishmania species and clinical traits of Cutaneous Leishmaniasis cases in the Colombian army. PLoS Negl Trop Dis. 2017;11(8):e0005876.

45. van der Meide WF, Jensema AJ, Akrum RA, Sabajo LO, Lai A Fat RF, Lambregts L, et al. Epidemiology of cutaneous leishmaniasis in Suriname: a study performed in 2006. Am J Trop Med Hyg; 2008;79(2):192-7.

46. Kato H, Gomez EA, Martini-Robles L, Muzzio J, Velez L, Calvopiña M, et al. Geographic Distribution of Leishmania Species in Ecuador Based on the Cytochrome B Gene Sequence Analysis. PLoS Negl Trop Dis. 2016;10(7):e0004844.

47. Souza AAA, Silveira FT, Lainson R, Barata IR, Silva MGS, Lima JAN, et al. The Phlebotominae fauna of Serra dos Carajás, Pará, Brazil, and its possible implication in the transmission of American tegumentary leishmaniasis. Rev Pan-Amaz Saude. 2010;1(1):45-51. 